Pacific Journal of Mathematics

THREE DIMENSIONAL HOMOGENEOUS ALGEBRAS 


\title{
THREE DIMENSIONAL HOMOGENEOUS ALGEBRAS
}

\author{
J. A. MacDougall and L. G. Sweet
}

An algebra $A$ is homogeneous if its automorphism group acts transitively on the set of one dimensional subspaces of $A$. In this paper the structure of all three dimensional homogeneous algebra is determined. These fall into three classes: (1) truncated quaternion algebras over formally real Pythagorean fields; (2) an algebra over GF(2) in which $x^{2}=x$ for all $x$ in $A$, and (3) two algebras over GF(2) which are generated by each of their nonzero elements. The automorphism group is determined in each case.

All algebras considered are assumed to be finite dimentional but not necessarily associative. If $A$ is an algebra we denote its group of algebra automorphisms by $\operatorname{Aut}(A)$. An algebra $A$ is said to be homogeneous if $\operatorname{Aut}(A)$ acts transitively on the set of one dimensional subspaces of $A$. The reader is referred to a paper by one of the authors [3] for a discussion of arbitrary homogeneous algebras and a bibliography of the related literature. The purpose of this paper is to determine the structure of all three dimensional homogeneous algebras.

Throughout this paper we assume that $A$ is a nonzero three dimensional homogeneous algebra. Such algebras can be divided into four types in the following way. Let $a$ be any nonzero element of $a$ and let $\langle a\rangle$ denote the algebra generated by $a$. Then the four types are as follows:

$$
\begin{array}{ll}
\text { Type 1. } & a^{2}=0 \\
\text { Type 2. } & a^{2}=\lambda a, \lambda \text { a nonzero scalar } \\
\text { Type 3. } & \operatorname{dim}\langle a\rangle=2 \\
\text { Type 4. } & \operatorname{dim}\langle a\rangle=3 .
\end{array}
$$

We now investigate each type separately.

Type 1. $a^{2}=0$.

Since $a^{2}=0$ the homogeneity condition implies that $x^{2}=0$ for all $x \in A$ and this implies that $A$ is anti-commutative. Clearly $A$ is not a quasi division algebra and so it follows from the results of Shult [1] and Gross [2] that the underlying field $K$ must be infinite.

Let $a$ be any nonzero element of $A$. Suppose we can find a nonzero $b \in A, b \neq \lambda a$ such that

$$
a b=\lambda_{1} a+\lambda_{2} b .
$$


If $\lambda_{2}=0$ then $\operatorname{tr} L_{a}=0$ (see [3]) implies that $L_{a}$ is nilpotent. But then the homogeneity condition implies that $L_{x}$ and $R_{x}$ are nilpotent for all $x$ and so $A$ is a special nil algebra. Since $K$ is infinite we may use Theorem 2 of [4] to conclude that $A^{2}=0$. If $\lambda_{2} \neq 0$ then extend $\{a, a b\}$ to a basis for $A$. It is now easy to contradict the fact that $L_{a}$ and $L_{a b}$ must be projectively similar. Hence $a b$ never depends on $a$ and $b$ when $a$ and $b$ are independent.

Now choose a basis $a, b, a b$ for $A$. Then

$$
L_{a}=\left[\begin{array}{llc}
0 & 0 & \alpha_{1} \\
0 & 0 & \alpha_{2} \\
0 & 1 & 0
\end{array}\right] \quad L_{b}=\left[\begin{array}{rcc}
0 & 0 & \beta_{1} \\
0 & 0 & \beta_{2} \\
-1 & 0 & 0
\end{array}\right] \quad L_{a b}=\left[\begin{array}{ccc}
-\alpha_{1} & -\beta_{1} & 0 \\
-\alpha_{2} & -\beta_{2} & 0 \\
0 & 0 & 0
\end{array}\right] \text {. }
$$

If necessary we can choose a new $b$ to force $\alpha_{1}=0$ and then $\operatorname{tr} L_{a b}=0$ implies that $\beta_{2}=0$. So we assume we have a basis $a, b$ and $a b$ such that

$$
L_{a}=\left[\begin{array}{lll}
0 & 0 & 0 \\
0 & 0 & \alpha \\
0 & 1 & 0
\end{array}\right] \quad L_{b}=\left[\begin{array}{rrr}
0 & 0 & \beta \\
0 & 0 & 0 \\
-1 & 0 & 0
\end{array}\right] \quad L_{a b}=\left[\begin{array}{rrr}
0 & -\beta & 0 \\
-\alpha & 0 & 0 \\
0 & 0 & 0
\end{array}\right]
$$

If $\alpha=0$ then the fact that $L_{a}$ and $L_{b}$ are projectively similar forces $\beta=0$ and then $L_{a b}=0$ which is impossible. Hence $\alpha \neq 0$ and similarly $\beta \neq 0$.

We now show that $A$ is a homogeneous algebra under the following conditions:

(i) $-\alpha$ is a nonzero square in $K$.

(ii) $\beta$ is a nonzero square in $K$.

(iii) $K$ has the property that the sum of nonzero squares is always a nonzero square (such a field is called a formally real Pythagorean field).

Let $\sigma \in \operatorname{Aut}(A)$. By considering $\sigma(x y)=\sigma(x) \sigma(y)$ as $x$ and $y$ run through the basis $a b, a, b$ it is easy to show that

$$
\sigma=\left[\begin{array}{rrr}
C_{11} & -\beta / \alpha C_{21} & \beta C_{13} \\
-\alpha / \beta C_{21} & C_{22} & -\alpha C_{23} \\
1 / \beta C_{31} & -1 / \alpha C_{31} & C_{33}
\end{array}\right]
$$

where $C_{i j}$ is the cofactor of the $i j$ entry. Conversely any invertible matrix of this form represents an automorphism of $A$. It remains to be shown under what conditions Aut $(A)$ actually acts transitively on the one dimensional subspaces of $A$. By considering compositions of automorphisms it is easy to see that $A$ is homogeneous if and only if there exists a $\sigma \in \operatorname{Aut}(A)$ such that $\sigma(a)=\gamma\left(\lambda_{1} a+\lambda_{2} b+\lambda_{3} a b\right)$ for any nonzero triple $\left(\lambda_{1}, \lambda_{2}, \lambda_{3}\right)$. If such an automorphism exists 
then $\sigma L_{a}=\gamma L_{\lambda_{1} a+\lambda_{2} b+\lambda_{3} a b} \sigma$ and this implies that

$$
-\alpha=\gamma^{2}\left(-\alpha \lambda_{1}^{2}+\beta \lambda_{2}^{2}-\beta \alpha \lambda_{3}^{2}\right) .
$$

Since $\alpha \neq 0$ this equation forces conditions (1), (2), and (3). On the other hand suppose conditions (1), (2), and (3) are true. We wish to construct an automorphism $\sigma$ of the form

$$
\sigma=\left[\begin{array}{lll}
\gamma \lambda_{1} & x_{1} & x_{2} \\
\gamma \lambda_{2} & x_{3} & x_{4} \\
\gamma \lambda_{3} & x_{5} & x_{6}
\end{array}\right]=\left[\begin{array}{rrr}
C_{11} & -\beta / \alpha C_{12} & \beta C_{13} \\
-\alpha / \beta C_{21} & C_{22} & -\alpha C_{23} \\
1 / \alpha C_{31} & -1 / \alpha C_{32} & C_{33}
\end{array}\right]
$$

This gives us a homogeneous linear system of the form $B x=0$ where $x=\left(x_{1}, x_{2}, x_{3}, x_{4}, x_{5}, x_{6}\right)$ and

$$
B=\left[\begin{array}{cccccc}
1 & 0 & 0 & \beta / \alpha \gamma \lambda_{3} & 0 & -\beta / \alpha \gamma \lambda_{2} \\
0 & 1 & \beta \gamma \lambda_{3} & 0 & -\beta \gamma \lambda_{2} & 0 \\
0 & \gamma \lambda_{3} & 1 & 0 & 0 & -\gamma \lambda_{1} \\
\alpha \gamma \lambda_{3} & 0 & 0 & 1 & -\alpha \gamma \lambda_{1} & 0 \\
0 & \gamma / \alpha \lambda_{2} & 0 & -\gamma / \alpha \lambda_{1} & 1 & 0 \\
\gamma \lambda_{2} & 0 & -\gamma \lambda_{1} & 0 & 0 & 1
\end{array}\right]
$$

and also another system of quadratic equations

$$
\begin{aligned}
& \gamma \lambda_{1}=x_{3} x_{6}-x_{4} x_{5} \\
& \gamma \lambda_{2}=\frac{\alpha}{\beta}\left(x_{1} x_{6}-x_{2} x_{5}\right) \\
& \gamma \lambda_{3}=\frac{1}{\beta}\left(x_{1} x_{4}-x_{2} x_{3}\right) .
\end{aligned}
$$

If $\lambda_{1}=\lambda_{2}=0$ then it can be checked that $x_{1}=0, x_{2}=-\beta \gamma \lambda_{3}$, $x_{3}=1, x_{4}=0, x_{5}=0, x_{6}=0$ is a solution of both systems if we take $\gamma=1 / \sqrt{u} \lambda_{3}$. Suppose $\lambda_{1}$ and $\lambda_{2}$ are not both zero. Let $d=$ $\sqrt{-\alpha \lambda_{1}^{2}+\beta \lambda_{2}^{2}}$. Then it can be checked that

$$
x_{1}=\frac{\beta \gamma \lambda_{2}}{\alpha d}, \quad x_{1}=\frac{-\beta \gamma^{2} \lambda_{1} \lambda_{3}}{d}, \quad x_{3}=\frac{\gamma \lambda_{1}}{d}, \quad x_{4}=\frac{-\beta \gamma^{2} \lambda_{2} \lambda_{3}}{d},
$$

$x_{5}=0, x_{6}=d$ is a solution of both systems if we take

$$
\gamma=\sqrt{\frac{-\alpha}{-\alpha \lambda_{1}^{2}+\beta \lambda_{2}^{2}-\alpha \beta_{3}^{2}}} .
$$

Hence $A$ is homogeneous.

So if $A$ is a three dimensional homogeneous algebra of Type 1 we can choose a basis $a, b, a b$ so that the multiplication table becomes 


\begin{tabular}{r|rrr} 
& $a$ & $b$ & $a b$ \\
\hline$a$ & 0 & $a b$ & $\alpha b$ \\
$b$ & $-a b$ & 0 & $\beta a$ \\
$a b$ & $-\alpha b$ & $-\beta a$ & 0
\end{tabular}

where

(i) $-\alpha$ is a nonzero square in $K$.

(ii) $b$ is a nonzero square in $K$.

(iii) $K$ is a formally real Pythagorean field.

These algebras are related to the so-called quaternion algebras. Let $K$ be any field and $V$ be a 4-dimensional vector space over $K$ with basis $1, x_{1}, x_{2}, x_{3}$. Now define a multiplication on $V$ by using the following table where $\alpha, \beta$ are any nonzero scalars

\begin{tabular}{|c|c|c|c|c|}
\hline & 1 & $x_{1}$ & $x_{2}$ & $x_{3}$ \\
\hline 1 & 1 & $x_{1}$ & $x_{2}$ & $x_{3}$ \\
\hline$x_{1}$ & $x_{1}$ & $\alpha 1$ & $x_{3}$ & $\alpha x_{2}$ \\
\hline$x_{2}$ & $x_{2}$ & $-x_{3}$ & $\beta 1$ & $-\beta x_{1}$ \\
\hline$x_{3}$ & $x_{3}$ & $-\alpha x_{2}$ & $\beta x_{1}$ & $-\alpha \beta 1$ \\
\hline
\end{tabular}

Then $V$ is called a quaternion algebra with parameters $\alpha$ and $\beta$. We can now define a 3-dimensional algebra over $K$ by deleting the top row, the left-most column and replacing the main diagonal of the above table with zeros. The resulting algebra is called the truncated algebra of pure quaternions.

We have shown that if $A$ is a 3-dimensional homogeneous algebra of Type 1 over a field $K$ then $A$ is a truncated quaternion algebra with parameters $\alpha,-\beta$ where $-\alpha$ and $\beta$ are nonzero squares and $K$ is formally real Pythagorean field. It is interesting to note that all such algebras over a given field are actually isomorphic. In particular consider $A_{1}$, the usual vector cross product with basis $i, j, k$ where $i j=-j i=k, i k=-k i=-j, j k=-k j=i, i^{2}=j^{2}=$ $k^{2}=0$. Suppose $A_{2}$ is a homogeneous algebra with $a b=-b a=c$, $a c=-c a=\alpha b, b c=-c b=\beta a, a^{2}=b^{2}=c^{2}=0$ where $-\alpha$ and $\beta$ are squares. Define a linear map $\sigma: A_{2} \rightarrow A_{1}$, by extending $\sigma(\alpha)=$ $-\sqrt{-\alpha} i, \sigma(b)=\sqrt{\beta} j$ and $\sigma(c)=-\sqrt{-\alpha \beta} k$. Then it is easily checked that $\sigma$ is an algebra isomorphism. So we have shown that a 3dimensional homogeneous algebra of Type 1 is isomorphic to the usual vector cross product algebra over a formally real Pythagorean field $K$.

Type 2. $a^{2}=\lambda a, \lambda$ a nonzero scalar.

In this case the homogeneity condition implies that $x^{2}=\lambda x$ for 
all $x \in A$ where $\lambda$ is a nonzero scalar which may depend on $x$. Clearly $A$ must be power associative. It was shown in [3] that $K$ must be $\mathrm{GF}(2)$. But Gross showed [1] that the only nonzero homogeneous algebras over $\mathrm{GF}(2)$ are always commutative, quasi division algebras.

Choose $a, b \in A$ with $b \neq a$. Then $a b \neq a$ and $a b \neq b$. Also since $\operatorname{tr} L_{a}=0$ but $\operatorname{det} L_{a}=1$ it follows that $a b \neq a+b$. Hence $a, b, a b$ form a basis for $A$. With respect to this basis we have

$$
L_{a}=\left[\begin{array}{ccc}
1 & 0 & \alpha_{1} \\
0 & 0 & \alpha_{2} \\
0 & 1 & 1
\end{array}\right] \quad L_{b}=\left[\begin{array}{ccc}
0 & 0 & \beta_{1} \\
0 & 1 & \beta_{2} \\
1 & 0 & 1
\end{array}\right] \quad L_{a b}=\left[\begin{array}{ccc}
\alpha_{1} & \beta_{1} & \gamma_{1} \\
\alpha_{2} & \beta_{2} & \gamma_{2} \\
1 & 1 & \alpha_{1}+\beta_{2}
\end{array}\right] .
$$

Since $\operatorname{det} L_{a}=\operatorname{det} L_{b}=1$ we must have $\alpha_{2}=\beta_{1}=1$. If necessary replace $b$ by $a+b$ to force $\alpha_{1}=1$. Since $\operatorname{det} L_{a b}=1$ we must have $\beta_{2}=0$. Also $\operatorname{det}\left(L_{a}+L_{a b}\right)=1$ forces $\gamma_{2}=0$ and finally $\operatorname{det}\left(L_{b}+\right.$ $\left.L_{a b}\right)=1$ forces $\gamma_{1}=0$.

Hence $A$ is of the form

\begin{tabular}{r|ccc} 
& $a$ & $b$ & $a b$ \\
\hline$a$ & $a$ & $a b$ & $a+b+a b$ \\
$b$ & $a b$ & $b$ & $a+a b$ \\
$a b$ & $a+b+a b$ & $a+a b$ & $a b$
\end{tabular}.

It is easily checked that $A$ is a homogeneous algebra. In fact Aut $(A)$ is the group generated by

$$
\left[\begin{array}{lll}
0 & 1 & 0 \\
1 & 1 & 1 \\
0 & 0 & 1
\end{array}\right] \text { and }\left[\begin{array}{lll}
0 & 0 & 1 \\
1 & 1 & 1 \\
0 & 1 & 1
\end{array}\right]
$$

Type 3. $\operatorname{dim}\langle a\rangle=2$.

In this case $\langle a\rangle$ is a 2-dimensional subalgebra for each nonzero $a$ in $A$. Fix $a \in A-(0)$ and choose $b \in A-\langle a\rangle$. Then $\langle a\rangle$ and $\langle b\rangle$ are two distinct 2-dimensional subalgebras of the 3 -dimensional subalgebra $A$ and so $\langle a\rangle \cap\langle b\rangle$ is a 1-dimensional subalgebra $\langle c\rangle$, contradicting the first line of this paragraph. Hence there are no three dimensional homogeneous algebras of Type 3.

Type 4. $\operatorname{dim}\langle a\rangle=3$.

In this case we have $\langle a\rangle=A$. We first assume that $A$ is commutative. The either $a, a^{2}, a a^{2}$ or $a, a^{2}, a^{2} a^{2}$ must form a basis. We consider the two cases separately.

(a) Suppose $a, \alpha^{2}, a \alpha^{2}$ do not form a basis. Then $a a^{2}=\gamma_{1} a+\gamma_{2} a^{2}$ and $a, a^{2}, a^{2} a^{2}$ does form a basis. The homogeneity condition now 
implies that for any $\lambda \in K$

$$
\left(a+\lambda a^{2}\right)\left(a+\lambda a^{2}\right)^{2}=\gamma_{1}^{\prime}\left(a+\lambda a^{2}\right)+\gamma_{2}^{\prime}\left(a+\lambda a^{2}\right)^{2} \text { for some } \gamma_{1}^{\prime}, \gamma_{2}^{\prime} \in K .
$$

Simplifying and comparing coefficients with respect to the basis $a, a^{2}, a^{2} a^{2}$ we get the following system

$$
\begin{aligned}
\gamma_{1}^{\prime}+\gamma_{2}^{\prime}\left(2 \lambda \gamma_{1}\right) & =\gamma_{1}+\lambda\left(2 \gamma_{1} \gamma_{2}\right)+\lambda^{2}\left(\alpha_{1}+2 \gamma_{1}^{2}\right)+\lambda^{3} \beta_{1} \\
\gamma_{1}^{\prime} \lambda+\lambda_{2}^{\prime}\left(1+2 \lambda \gamma_{2}\right) & =\gamma_{2}+\lambda\left(2 \gamma_{1}+2 \gamma_{2}^{2}\right)+\lambda^{2}\left(\alpha_{2}+2 \gamma_{1} \gamma_{2}\right)+\lambda^{3} \beta_{2} \\
\gamma_{2}^{\prime} \lambda^{2} & =\lambda+\lambda^{2}\left(-\gamma_{2}+2 \gamma_{2}\right)+\lambda^{3}\left(-\gamma_{1}\right) .
\end{aligned}
$$

Now solving the first two equations for $\gamma_{2}^{\prime}$ and comparing to the third equation gives us

$$
\begin{array}{r}
\lambda+\lambda^{2}\left(2 \gamma_{2}\right)+\lambda^{3}\left(-4 \gamma_{1}\right)+\lambda^{4}\left(-4 \gamma_{1} \gamma_{2}-\alpha_{2}\right)+\gamma^{5}\left(4 \gamma_{1}^{2}-\beta_{2}+\alpha_{1}\right)-\lambda^{6} \beta_{1}=0 \\
\text { for all } \lambda \in K .
\end{array}
$$

This implies that the field is finite and so we know that $K=\mathrm{GF}(2)$ and $A$ is a commutative quasi division algebra. Now with respect to the basis $a, a^{2}, a^{2} a^{2}$

$$
L_{a}=\left[\begin{array}{ccc}
0 & 1 & \alpha_{1} \\
1 & 1 & \alpha_{2} \\
0 & 0 & 1
\end{array}\right] \quad L_{a^{2}}=\left[\begin{array}{ccc}
1 & 0 & \beta_{1} \\
1 & 0 & \beta_{2} \\
0 & 1 & 1
\end{array}\right] \quad L_{a^{2} a^{2}}=\left[\begin{array}{ccc}
\alpha_{1} & \beta_{1} & \xi_{1} \\
\alpha_{2} & \beta_{2} & \xi_{2} \\
1 & 1 & \alpha_{1}+\beta_{2}
\end{array}\right] \text {. }
$$

Now the fact that $L_{a}$ and $L_{a^{2}}$ are similar implies that $\beta_{2}=1$. Also $\operatorname{det} L_{a^{2}}=\operatorname{det}\left(L_{a}+L_{a^{2}}\right)=\operatorname{det}\left(L_{a^{2}}+L_{a^{2} a^{2}}\right)=1$ implies that $\beta_{1}=0$, $\alpha_{2}=0$ and $\xi_{1}=1$ respectively. Finally $\xi_{2}=\alpha_{1}=0$ because $L_{a^{2} a^{2}}$ and $L_{a}+L_{a^{2} a^{2}}$ are similar to $L_{a}$. It follows that $A$ has a basis $a, a^{2}, a^{2} a^{2}$ with the following multiplication table:

\begin{tabular}{c|ccc} 
& $a$ & $a^{9}$ & $a^{2} a^{2}$ \\
\hline$a$ & $a^{2}$ & $a+a^{2}$ & $a^{2} \alpha^{2}$ \\
$a^{2}$ & $a+a^{2}$ & $a^{2} a^{2}$ & $a^{2}+a^{2} a^{2}$ \\
$a^{2} a^{2}$ & $a^{2} a^{2}$ & $a^{2}+a^{2} a^{2}$ & $a+a^{2} a^{2}$
\end{tabular}.

It is easily checked that $A$ is indeed a homogeneous algebra. In fact $\operatorname{Aut}(A)$ is a cyclic group of order seven generated by

$$
\sigma=\left[\begin{array}{lll}
0 & 0 & 1 \\
1 & 0 & 0 \\
0 & 1 & 1
\end{array}\right]
$$

(b) Suppose $a, a^{2}, a a^{2}$ do form a basis. If $K$ is finite then $K=\mathrm{GF}(2)$ and $A$ must be a quasi division algebra. In fact it follows from the papers of Gross [1] and Shult [2] that the characteristic 
polynomial of $L_{a}$ must be $x^{3}+1$. Then with respect to our basis $a, a^{2}, a a^{2}$ we have

$$
L_{a}=\left[\begin{array}{lll}
0 & 0 & 1 \\
1 & 0 & 0 \\
0 & 1 & 0
\end{array}\right] \quad L_{a^{2}}=\left[\begin{array}{lll}
0 & \beta_{1} & \gamma_{1} \\
0 & \beta_{2} & \gamma_{2} \\
1 & \beta_{3} & \gamma_{3}
\end{array}\right] \quad L_{a a^{2}}=\left[\begin{array}{ccc}
1 & \gamma_{1} & \xi_{1} \\
0 & \gamma_{2} & \xi_{2} \\
0 & \beta_{2} & 1+\gamma_{2}
\end{array}\right] .
$$

Now the equations $\operatorname{det}\left(\gamma_{1} L_{a}+L_{a^{2}}\right)=\operatorname{det}\left(\beta_{2} L_{a}+L_{a a^{2}}\right)=\operatorname{det} L_{a^{2}}=\operatorname{det} L_{a a^{2}}=$ $\operatorname{det}\left(L_{a^{2}}+L_{a a^{2}}\right)=1$ imply that $\beta_{1}=1+\gamma_{2}=\left(\beta_{2}=\gamma_{1}\right)=\left(\beta_{2}=\xi_{2}\right)=\left(\beta_{3}+\xi_{1}\right)=1$ respectively. Since $A$ is a homogeneous quasi division algebra generated by each of its elements we know that each automorphism (except the identity) is fixed point free. Consider the automorphism $\sigma$ for which $\sigma(\alpha)=a^{2}$. It is easily checked that this automorphism has an eigenvalue if $\beta_{3}=0$. So we must have $\beta_{3}=1$ and $\xi_{1}=0$. It follows that $A$ has a basis $a, a^{2}, a a^{2}$ with the multiplication table:

\begin{tabular}{c|ccc} 
& $a$ & $a^{2}$ & $a a^{2}$ \\
\hline$a$ & $a^{2}$ & $a a^{2}$ & $a$ \\
$a^{2}$ & $a a^{2}$ & $a+a^{2}+a a^{2}$ & $a+a a^{2}$ \\
$a a^{2}$ & $a$ & $a+a a^{2}$ & $a^{2}+a a^{2}$
\end{tabular}.

It is easily checked that this is a homogeneous algebra. In fact Aut $(A)$ is a cyclic group of order 7 generated by

$$
\sigma=\left[\begin{array}{lll}
0 & 1 & 0 \\
1 & 1 & 1 \\
0 & 1 & 1
\end{array}\right]
$$

Now assume that $K$ is infinite. Then with respect to the basis $a, a^{2}, a a^{2}$ we have

$$
L_{a}=\left[\begin{array}{ccc}
0 & 0 & \alpha_{1} \\
1 & 0 & \alpha_{2} \\
0 & 1 & 0
\end{array}\right] \quad L_{a^{2}}=\left[\begin{array}{ccc}
0 & \beta_{1} & \gamma_{1} \\
0 & \beta_{2} & \gamma_{2} \\
1 & \beta_{3} & -\beta_{2}
\end{array}\right] \quad L_{a a^{2}}=\left[\begin{array}{ccc}
\alpha_{1} & \gamma_{1} & \xi_{1} \\
\alpha_{2} & \gamma_{2} & \xi_{2} \\
0 & -\beta_{2} & -\alpha_{1}-\gamma_{2}
\end{array}\right] .
$$

Suppose $\alpha_{1}=0$. Then $\operatorname{det}\left(\lambda_{1} L_{a}+\lambda_{2} L_{a^{2}}+\lambda_{3} L_{a a^{2}}\right)=0$ for all $\lambda_{1}, \lambda_{2}, \lambda_{3} \in K$. But

$$
\begin{aligned}
\operatorname{det} & \left(\lambda_{1} L_{a}+\lambda_{2} L_{a^{2}}+\lambda_{3} L_{a a^{2}}\right)=\lambda_{1}^{2} \lambda_{2} \gamma_{1}+\lambda_{1}^{2} \lambda_{3} \xi_{1}+\lambda_{1} \lambda_{2}^{2}\left(\beta_{3} \gamma_{1}+\beta_{1} \beta_{2}+\beta_{1} \alpha_{2}\right) \\
& +\lambda_{1} \lambda_{2} \lambda_{3}\left(2 \alpha_{2} \gamma_{1}+\beta_{1} \alpha_{2}+\beta_{3} \xi_{1}\right)+\lambda_{1} \lambda_{3}^{2}\left(\gamma_{1} \gamma_{2}-\beta_{2} \xi_{1}+\alpha_{2} \xi_{1}\right) \\
& +\lambda_{2}^{2} \lambda_{3}\left(\alpha_{2} \beta_{3} \gamma_{1}+\alpha_{2} \beta_{1} \beta_{2}+\beta_{1} \xi_{2}-\beta_{2} \xi_{1}\right)+\lambda_{2} \lambda_{3}^{2}\left(\alpha_{2} \beta_{1} \gamma_{2}+\alpha_{2} \beta_{3} \xi_{1}+\xi_{2} \gamma_{1}-\gamma_{2} \xi_{1}\right) \\
& +\lambda_{3}^{3} \alpha_{2}\left(\gamma_{1} \gamma_{2}-\beta_{2} \xi_{1}\right)+\lambda_{2}^{3}\left(\beta_{1} \gamma_{2}-\beta_{2} \gamma_{1}\right)=0 .
\end{aligned}
$$

Since $K$ is infinite this implies that all the coefficients must be zero. It follows that $\gamma_{1}=\xi_{1}=\beta_{1}\left(\beta_{2}+\alpha_{2}\right)=\beta_{1} \gamma_{2}=0$. If $\beta_{1}=0$ the equa- 
tion $x y=a$ has no solution which is impossible in a nonzero homogeneous algebra. Hence $\beta_{1} \neq 0$ and $\beta_{2}=-\alpha_{2}$. If $\alpha_{1}=0$, then rank $L_{a}=2$ but rank $\left(L_{a a^{2}}-\alpha_{2} L_{a}\right)<2$ which is impossible. We conclude that $\alpha_{1} \neq 0$ and so $A$ must be a quasi division algebra. Since $\langle a\rangle=A$ we know that no automorphism of $A$ (except the identity) can have and eigenvalue.

Now consider the automorphism $\sigma$ for which $\sigma(a)=\mu\left(a+\lambda \alpha^{2}\right)$ where $\lambda$ is arbitrary and $\mu$ may depend on $\lambda$. Then it can be checked that

$$
\sigma=\left[\begin{array}{lll}
\mu & \mu^{2} \lambda^{2} \beta_{1} & \mu^{3}\left(\lambda\left(\beta_{1}+2 \alpha_{1}\right)+\lambda^{2}\left(\beta_{3} \alpha_{1}+2 \gamma_{1}\right)+\lambda^{3}\left(\beta_{1} \beta_{2}+\beta_{3} \gamma_{1}\right)\right) \\
\mu \lambda & \mu^{2}\left(1+\lambda^{2} \beta_{2}\right) & \mu^{3}\left(\lambda\left(\beta_{2}+2 \alpha_{2}\right)+\lambda^{2}\left(\beta_{1}+\beta_{3} \alpha_{2}+2 \gamma_{2}\right)+\lambda^{3}\left(\beta_{2}^{2}+\beta_{3} \gamma_{2}\right)\right) \\
0 & \mu^{2}\left(2 \lambda+\lambda^{2} \beta_{2}\right) & \mu^{3}\left(1+\lambda \beta_{3}-\lambda^{2} \beta_{2}+\lambda^{3} \beta_{1}\right)
\end{array}\right] .
$$

Suppose char $K \neq 2$. If $\beta_{3} \neq 0$ then letting $\lambda=-2 / \beta_{3}$ gives $\sigma$ an eigenvalue which is impossible. Hence we must have $\beta_{3}=0$. But then consider the automorphism $\tau$ for which $\tau(a)=\nu a^{2}$. It is easily checked that $\tau$ has an eigenvalue. Hence it follows that char $K=2$.

We now consider $\sigma\left(a^{2} a^{2}\right)=\sigma\left(a^{2}\right) \sigma\left(a^{2}\right)$. This gives us a system of 3 equations which can be solved to get

$$
\begin{aligned}
1+\lambda & \beta_{3}+\lambda^{4}\left(\beta_{1} \beta_{3}+\beta_{3}^{2} \alpha_{2}+\beta_{2}^{2}\right)+\lambda^{5} \beta_{3}\left(\beta_{2}^{2}+\beta_{3} \gamma_{2}+\beta_{3} \alpha_{1}\right) \\
& +\lambda^{6}\left(\beta_{1}^{2}+\beta_{1} \beta_{2} \beta_{3}+\beta_{3}^{2} \gamma_{1}\right) \\
= & \mu\left[1+\lambda^{4}\left(\beta_{2}^{2}+\beta_{3} \alpha_{1}+\beta_{3} \gamma_{2}\right)+\lambda^{6}\left(\beta_{1}^{2}+\beta_{3}^{2} \xi_{2}+\beta_{2} \beta_{3} \alpha_{1}+\beta_{2} \beta_{3} \gamma_{2}\right)\right. \\
& \left.+\lambda^{7}\left(\beta_{3}^{2} \xi_{1}+\beta_{1} \beta_{3} \alpha_{1}+\beta_{1} \beta_{3} \gamma_{2}\right)\right]
\end{aligned}
$$

or $f=\mu g$ where $f$ and $g$ are polynomials $\lambda$. Squaring we have $f^{2}=\mu^{2} g^{2}$. But since $\sigma(\alpha)=\mu\left(\alpha+\lambda a^{2}\right)$ we have $\alpha_{2}=\mu^{2} h$ where $h=$ $\alpha_{2}+\lambda\left(\beta_{1}+\alpha_{1}+\gamma_{2}+\beta_{3} \alpha_{2}\right)+\lambda^{2}\left(\gamma_{1}+\beta_{2}^{2}+\beta_{3} \gamma_{2}\right)$ and so comparing we have

$$
\alpha_{2} g^{2}=f^{2} h
$$

and so

$$
\begin{aligned}
& \alpha_{2}\left(1+\lambda^{8} *+\lambda^{12} *+\lambda^{14} *\right)=\left(1+\lambda^{2} \beta_{3}+\lambda^{8} *+\lambda^{10} *+\lambda^{12} *\right) \\
& \times\left(\alpha_{2}+\lambda\left(\beta_{1}+\alpha_{1}+\gamma_{2}+\beta_{3} \alpha_{2}\right)+\lambda^{2}\left(\alpha_{1}+\beta_{2}^{2}+\beta_{3} \gamma_{2}\right)\right)
\end{aligned}
$$

Since the field is infinite and $\lambda$ is arbitrary all the coefficients of $\lambda$ must be zero. Considering the coefficients of $\lambda, \lambda^{4}$, and $\lambda^{2}$ in that order we conclude that

$$
\begin{aligned}
\beta_{1}+\alpha_{1}+\gamma_{2}+\beta_{3} \alpha_{2} & =0 \\
\gamma_{1}+\beta_{2}^{2}+\beta_{3} \alpha_{2} & =0 \\
\alpha_{2} & =0 .
\end{aligned}
$$


Now $\alpha_{2}=0$ and since $A$ is a quasi division algebra we may without loss of generality assume that $L_{a}$ has an eigenvalue of 1 . That is, we may assume that $\alpha_{1}=1$. But again since $\sigma(a)=\mu\left(a+\lambda a^{2}\right)$ we conclude that

$$
1=\mu^{3}\left(1+\lambda\left(\gamma_{1}+\beta_{3}\right)+\lambda^{2}\left(\beta_{1} \beta_{2}+\beta_{3} \gamma_{1}+\beta_{2}\right)+\lambda^{3}\left(\beta_{1} \gamma_{2}+\beta_{2} \gamma_{1}\right)\right)
$$

or

$$
1=\mu^{3} k \text {. }
$$

From above we have $f^{3}=\mu^{3} g^{3}$ and so comparing we have

$$
f^{3} k=g^{3} .
$$

That is

$$
\begin{gathered}
\left(1+\lambda \beta_{3}+\lambda^{2} \beta_{3}^{2}+\lambda^{3} \beta_{3}^{3}+\cdots+\lambda^{18} *\right)\left(1+\lambda\left(\gamma_{1}+\beta_{3}\right)+\lambda^{2}\left(\beta_{1} \beta_{2}+\beta_{3} \gamma_{1}+\beta_{2}\right)\right. \\
\left.\quad+\lambda^{3}\left(\beta_{1} \gamma_{2}+\beta_{2} \gamma_{1}\right)\right)=1+\lambda^{4} *+\cdots+\lambda^{21} * .
\end{gathered}
$$

As before all the coefficients of $\lambda$ must be zero. Considering the coefficients of $\lambda$ and $\lambda^{2}$ we find that

$$
\gamma_{1}=\beta_{2}\left(\beta_{1}+1\right)=0 .
$$

Now if $\beta_{2}=0$ an above equation implies that $\beta_{3} \gamma_{2}=0$. If $\beta_{3}=0$ then $\sigma$ has an eigenvalue which in impossible. If $\gamma_{2}=0$ then $\operatorname{det} L_{a^{2}}=0$ which is impossible. Hence we must have $\beta_{2} \neq 0$ and $\beta_{1}=1$. But then $\beta_{1}+1+\gamma_{2}=0$ implies that $\gamma_{2}=0$ which again is impossible. Hence no such algebra exists over an infinite field.

We have determined all commutative homogeneous algebras of dimension 3 and Type 4 . Now let $A$ be any 3-dimensional homogeneous algebra of Type 4. Pass from $A$ to $A^{+}$. Then $A^{+}$is a commutative homogeneous algebra. Suppose $A^{+} \neq 0 . A^{+}$cannot be Type 1 since there are no nonzero commutative homogeneous algebras of Type 1. If $A^{+}$is of Type 2 we know that $K=\mathrm{GF}(2) . A^{+}$cannot be of Type 3 since there are no nonzero homogeneous algebras of Type 3. If $A^{+}$is of Type 4 we have just shown that $K=\mathrm{GF}(2)$. So either $A^{+}$is a zero algebra or $K=\mathrm{GF}(2)$. If $A^{+}$is a zero algebra then $A$ is anti-commutative. But then we have $2 \alpha^{2}=0$ and since $a^{2} \neq 0$ this implies that char $K=2$. But then $A$ is commutative and so again we have shown above that $K=\mathrm{GF}(2)$. Hence the only nonzero homogeneous algebras of Type 4 exist øver $K=\mathrm{GF}(2)$. In such cases we know that $A$ is a commutative quasi division algebra and so we have found all nonzero 3-dimensional homogeneous algebras of Type 4. That is, if $A$ is a nonzero 3-dimensional homogeneous algebra of Type 4 then either $A$ has a basis $a, a^{2}, a^{2} a^{2}$ such that 


\begin{tabular}{c|ccc} 
& $a$ & $a^{2}$ & $a^{2} a^{2}$ \\
\hline$a$ & $a^{2}$ & $a+a^{2}$ & $a^{2} a^{2}$ \\
$a^{2}$ & $a+a^{2}$ & $a^{2} a^{2}$ & $a^{2}+a^{2} a^{2}$ \\
$a^{2} a^{2}$ & $a^{2} a^{2}$ & $a+a^{2} a^{2}$ & $a+a^{2} a^{2}$
\end{tabular}

or $A$ has a basis $\alpha, a^{2}, a a^{2}$ such that

\begin{tabular}{c|ccc} 
& $\alpha$ & $a^{2}$ & $\alpha \alpha^{2}$ \\
\hline$\alpha$ & $\alpha^{2}$ & $a a^{2}$ & $\alpha$ \\
$a^{2}$ & $\alpha \alpha^{2}$ & $\alpha+\alpha^{2}+a a^{2}$ & $\alpha+a a^{2}$ \\
$\alpha^{2} \alpha^{2}$ & $\alpha$ & $\alpha+\alpha a^{2}$ & $\alpha^{2}+a a^{2}$
\end{tabular}

\section{REFERENCES}

1. F. Gross, Finite automorphic algebras over $G F(2)$, Proc. Amer. Math. Soc., 31 (1972), 10-14.

2. E. E. Shult, On Finite automorphic algebras, Illinois J. Math., 13 (1969), 625-653.

3. L. G. Sweet, Homogeneous algebras, Pacific J. Math., 59 (1975), 585-594.

4. - On the triviality of homogeneous algebras over an algebraically closed field, Proc. Amer. Math. Soc., 48 (1975), 321-324.

Received February 1, 1977 and in revised form July 21, 1977. The research by the authors was supported by a grant from the Senate Research Committee of U.P.E.I.

UNIVERSity of Prince EdWARd Island

Charlottetown, Prince Edward Island, Canada 


\section{PACIFIC JOURNAL OF MATHEMATICS}

\section{EDITORS}

RICHARD ARENS (Managing Editor)

University of California

Los Angeles, California 90024

C. W. Curtis

University of Oregon

Eugene, OR 97403

C. C. MOORE

University of California

Berkeley, CA 94720

\section{J. DUGUNDJI}

Department of Mathematics University of Southern California Los Angeles, California 90007

R. Finn AND J. Milgram Stanford University Stanford, California 94305

ASSOCIATE EDITORS
E. F. BECKENBACH

B. H. NeUMANN

F. WOLF

K. YoshidA

\section{SUPPORTING INSTITUTIONS}

UNIVERSITY OF BRITISH COLUMBIA UNIVERSITY OF SOUTHERN CALIFORNIA CALIFORNIA INSTITUTE OF TECHNOLOGY STANFORD UNIVERSITY UNIVERSITY OF CALIFORNIA MONTANA STATE UNIVERSITY UNIVERSITY OF TOKYO UNIVERSITY OF NEVADA, RENO UNIVERSITY OF UTAH NEW MEXICO STATE UNIVERSITY OREGON STATE UNIVERSITY UNIVERSITY OF OREGON

WASHINGTON STATE UNIVERSITY UNIVERSITY OF WASHINGTON OSAKA UNIVERSITY 


\section{Pacific Journal of Mathematics}

\section{Vol. 74, No. $1 \quad$ May, 1978}

Gerald Arthur Anderson, Computation of the surgery obstruction groups

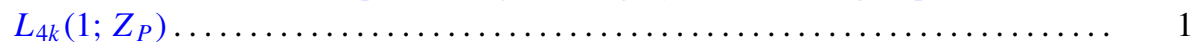

R. K. Beatson, The degree of monotone approximation ................ 5

Sterling K. Berberian, The character space of the algebra of regulated functions . . . 15

Douglas Michael Campbell and Jack Wayne Lamoreaux, Continua in the plane with

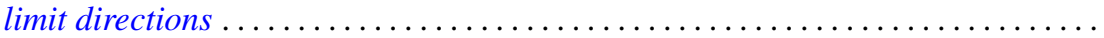

R. J. Duffin, Algorithms for localizing roots of a polynomial and the Pisot

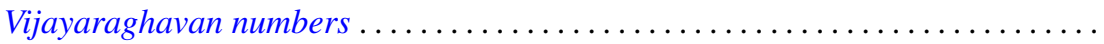

Alessandro Figà-Talamanca and Massimo A. Picardello, Functions that operate on

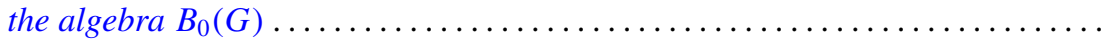

John Erik Fornaess, Biholomorphic mappings between weakly pseudoconvex

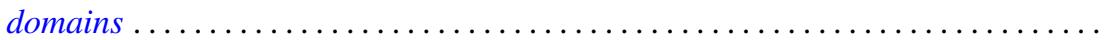

Andrzej Granas, Ronald Bernard Guenther and John Walter Lee, On a theorem of S.

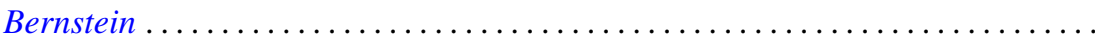

Jerry Grossman, On groups with specified lower central series quotients . .........

William H. Julian, Ray Mines, III and Fred Richman, Algebraic numbers, a constructive development . . . . . . . . . . . . . . . . . . . . . . .

Surjit Singh Khurana, A note on Radon-Nikodým theorem for finitely additive

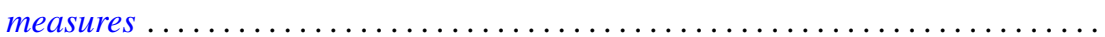

Garo K. Kiremidjian, A Nash-Moser-type implicit function theorem and nonlinear

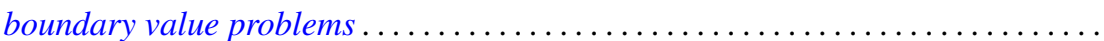

Ronald Jacob Leach, Coefficient estimates for certain multivalent functions ....

John Alan MacBain, Local and global bifurcation from normal eigenvalues. II . . 133

James A. MacDougall and Lowell G. Sweet, Three dimensional homogeneous algebras...

John Rowlay Martin, Fixed point sets of Peano continua ......

R. Daniel Mauldin, The boundedness of the Cantor-Bendixson order of some analytic sets...

Richard C. Metzler, Uniqueness of extensions of positive linear functions ..

Rodney V. Nillsen, Moment sequences obtained from restricted powers . .

Keiji Nishioka, Transcendental constants over the coefficient fields in differential elliptic function fields...

Gabriel Michael Miller Obi, An algebraic closed graph theorem

Richard Cranston Randell, Quotients of complete intersections by $\mathbf{C}^{*}$ actions . . 221

Bruce Reznick, Banach spaces which satisfy linear identities . .

Bennett Setzer, Elliptic curves over complex quadratic fields...

Arne Stray, A scheme for approximating bounded analytic functions on certain subsets of the unit disc.

Nicholas Th. Varopoulos, A remark on functions of bounded mean oscillation and bounded harmonic functions. Addendum to: "BMO functions and the

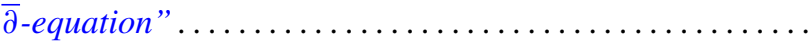

Charles Irvin Vinsonhaler, Torsion free abelian groups quasi-projective over their

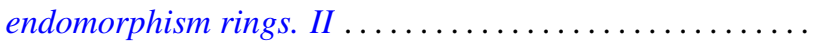

Thomas R. Wolf, Characters of $p^{\prime}$-degree in solvable groups ... 